Article

\title{
Restoration Plan for Degraded Forest in The Democratic People's Republic of Korea Considering Suitable Tree Species and Spatial Distribution
}

\author{
Sle-gee Lee ${ }^{1}$ (i), Hyun-Ah Choi ${ }^{2}$, Hyeji Yoo ${ }^{1}$, Cholho Song ${ }^{1}$ (D), Sungeun Cha ${ }^{1}$, Sang-Won Bae ${ }^{1}$, \\ Yowhan Son ${ }^{1}$ and Woo-Kyun Lee ${ }^{1, *}$ \\ 1 Department of Environmental Science and Ecological Engineering, Korea University, 145 Anam Ro, \\ Seoul 136-713, Republic of Korea; cristallize@naver.com (S.-g.L); oopshyeji@gmail.com (H.Y.); \\ cholhosong@gmail.com (C.S.); tgy2005@korea.ac.kr (S.C.); \\ kiefern82@gmail.com (S.-W.B.); yson@korea.ac.kr (Y.S.) \\ 2 Hanns Seidel Foundation Korea Office, Seoul 04419, Republic of Korea; hachoi@hss.or.kr \\ * Correspondence: leewk@korea.ac.kr
}

Received: 16 January 2018; Accepted: 14 March 2018; Published: 18 March 2018

\begin{abstract}
The ecosystem across the Democratic People's Republic of Korea (DPRK) is threatened by deforestation. However, there is very little attention being given to government efforts for afforestation and rehabilitation plan. The most significant barriers to addressing this problem are technique limitations, availability of information, and lack of a stepwise forest management plan. This study identifies spatially suitable tree species, and establishes a stepwise restoration plan to support decision making for restoring degraded forest in the DPRK throughout a suitable restoration map. First off, target species were chosen from reference data, and spatial distribution maps for each tree species were prepared based on social needs as well as natural conditions in the DPRK. The suitable restoration map was calculated by two priorities in a weighting method; suitable priority, and distributional clustering level. Finally, the 23 afforestation species were selected for the suitable restoration map, including 11 coniferous and 12 deciduous tree species. We introduced a stepwise afforestation/restoration plan of degraded forest in the DPRK; general (long-term), detailed (medium-term), implementation (short-term) plans. Maps with different spatial resolutions were prepared for each of the plans. A restoration map with $12.5 \mathrm{~km}$ spatial resolution can be used for the general plan at the national level, and maps with $5 \mathrm{~km}$ and $1 \mathrm{~km}$ spatial resolutions can be used for detailed plan at the local level and implementation plan at the site level, respectively.
\end{abstract}

Keywords: afforestation; degraded forest; DPRK; North Korea; spatial plant distribution; suitable tree species

\section{Introduction}

Deforestation in the Democratic People's Republic of Korea (DPRK) is not limited by national boundaries and threatens the entire Korean Peninsula's ecological health. Therefore, restoration of degraded forest has received consistent attention. In the last ten years, the DPRK government has noticed that existing implementation actions only have small outcomes, and therefore, they decided to concentrate on a restoration plan for the next ten years [1]. The government has relied on international support to overcome the current situation, for example, swidden-fallow agroforestry [2].

Forest area in the DPRK decreased from 9,171,700 ha in 1980 to 7,085,800 ha in 2000 [3], and pure forest area, which excludes degraded and barren ground, was estimated at 7,500,000 ha in 1999 [4]. In the meantime, severe degradation has progressed; denuded land has rapidly increased since 
the 1970s, with a 397\% rise between 1979 and 2010 [5]. There are various reasons that forest restoration efforts have been ineffective in the DPRK, but the most important problem has been rooted in limited forest management in difficult economic conditions [6].

Forest restoration fundamentally requires baseline data, for example, climate, topography, and soil conditions for identifying afforestation species and management criteria [7]; however, available open data is limited in the DPRK [8]. Another factor is the absence of a stepwise plan. Most planning in the DPRK is focused on the effectiveness for short-term forest resource utilization because there is little understanding of how the ecosystems work [9]. In contrast, South Korea has successfully restored degraded forest back to forest area, based on establishing long-term plans [10]. The successfully applied methodology and predicted data from the DPRK can be used to develop a stepwise restoration plan, long-term (general), medium-term (detailed), and short-term (implementation) plans. The aim of this research is to establish a stepwise plan to support policies that restore degraded forest in the DPRK, based on environmental information with multi-spatial resolution.

\section{Materials and Methods}

\subsection{Study Area and Data}

The spatial extent of this research in the DPRK includes 13 provinces (HHN: Hwanghaebuk-do, HHS: Hwanghaenam-do, GW: Gangwon-do, PAN: Pyeonganbuk-do, PAS: Pyeongannam-do, JG: Jagang-do, YG: Yanggang-do, HGN: Hamgyeongbuk-do, HGS: Hamhyeongnam-do) (Figure 1).

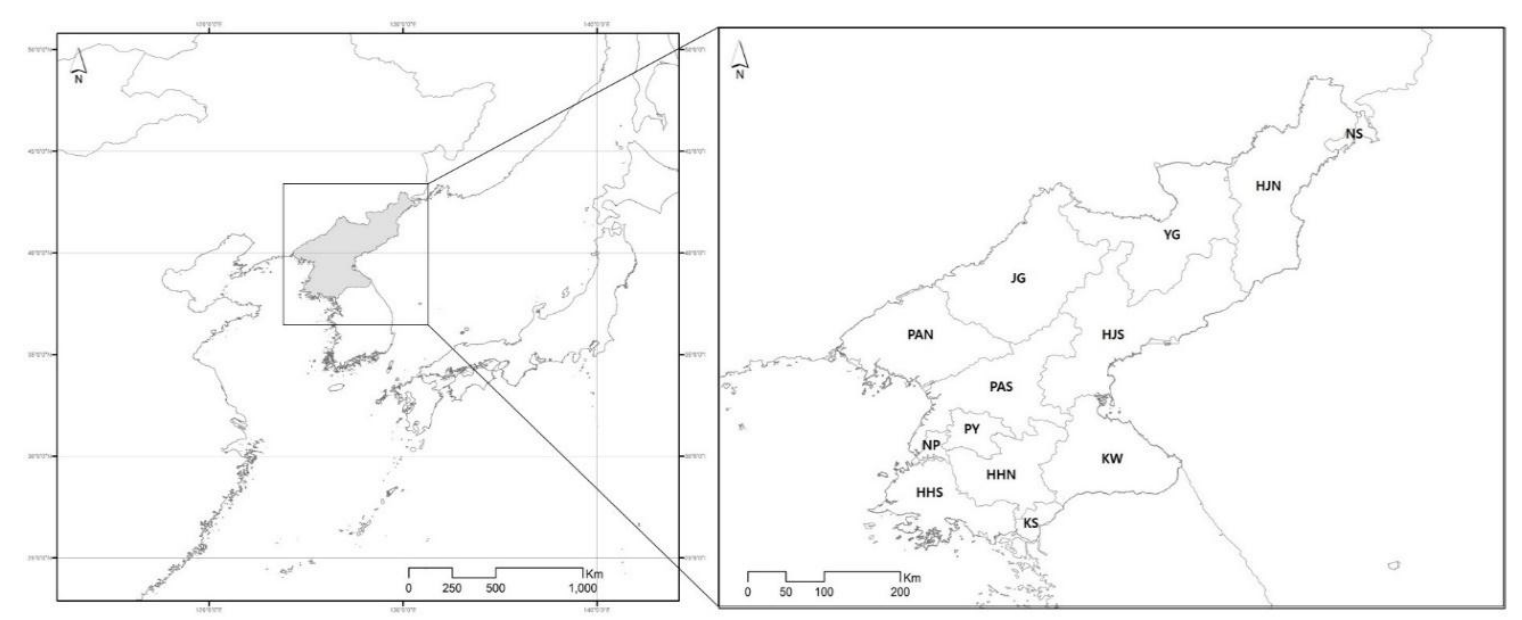

Figure 1. Location and administrative district of the study area in the Democratic People's Republic of Korea (DPRK).

The major data-sets were plant distribution and environmental data. Afforestation species data for selecting suitable tree species were based on the Tree Nursery Reference Book for Laborer [11]. Open source data was obtained from the National Agency plant database for South Korea [12-19], Korea National Arboretum [20], Korea Forest Service [21-23], and the Korean Academy of Science and Technology [24]. Environmental data included variables that affect plant growth and distribution. The selected spatial environmental information was categorized into climate, topography and soil factor. Climatic zone was used to classify the warmth index [25], and a $1 \mathrm{~km} \times 1 \mathrm{~km}$ spatial data for average temperature were obtained from the WorldClim database. Topographic variables consisted of physiographic division, elevation, aspect, and slope at a resolution of $200 \mathrm{~m} \times 200 \mathrm{~m}$, and were analyzed using geographic information system (GIS) software (Arcmap 10.1, ESRI, California, United States of America) [26].

Because the DPRK government recognizes land as an important source of production, they have consistently investigated the natural environments. In particular, soil is considered a base 
resource in agriculture and forestry, so the government has tried to carefully manage this resource [27]. However, data collected within the DPRK is often unavailable to foreign researchers, and even field investigations are not permitted. For this reason, this study used soil data based on Catena, which uses soil-landscape delineation [28]. Catena analysis is a three-dimensional division of soil landscape units developed to overcome the existing nine-unit landscape model. This technique has the advantage of applying qualitative pedogenic processes at the slope level. This study utilized $30 \mathrm{~m} \times 30 \mathrm{~m}$ predicted soil data for bed rock, soil texture, soil depth, topographic wetness index, organic matter and soil hardness $[29,30]$.

\subsection{Multi-Resolution Map and Stepwise Restoration Plan}

To prepare a stepwise restoration plan, suitable tree species were firstly selected for restoration, and its spatial distributions in degraded forest were identified considering natural and social needs in the DPRK. Then a suitable restoration map was prepared through priority analysis of suitability and cluster level. Finally, the stepwise restoration plan was made using multi-resolution maps.

\subsubsection{Assessing Degraded Forest in the DPRK}

Degraded forest in the DPRK was identified in a classification map from a TerraSAR-X image, obtained on 17 January 2014 [31]. This image divided degraded forest into a denuded area, as well as barren and cultivated land. The denuded land, which is covered by shrub or grass, can be classified as having a soft texture due to the absence of plant distribution, and it has shades of red similar to denuded land in images [32]. The classification map for degraded forest in the DPRK shows that deforestation has had a devastating impact on the entire area (Figure 2). The rate of forest degradation is more serious than agricultural area changes, which has been generally and traditionally used as crop land. Moreover, the denuded area is widely spread, corresponding to the indiscriminate land use policies in the DPRK.

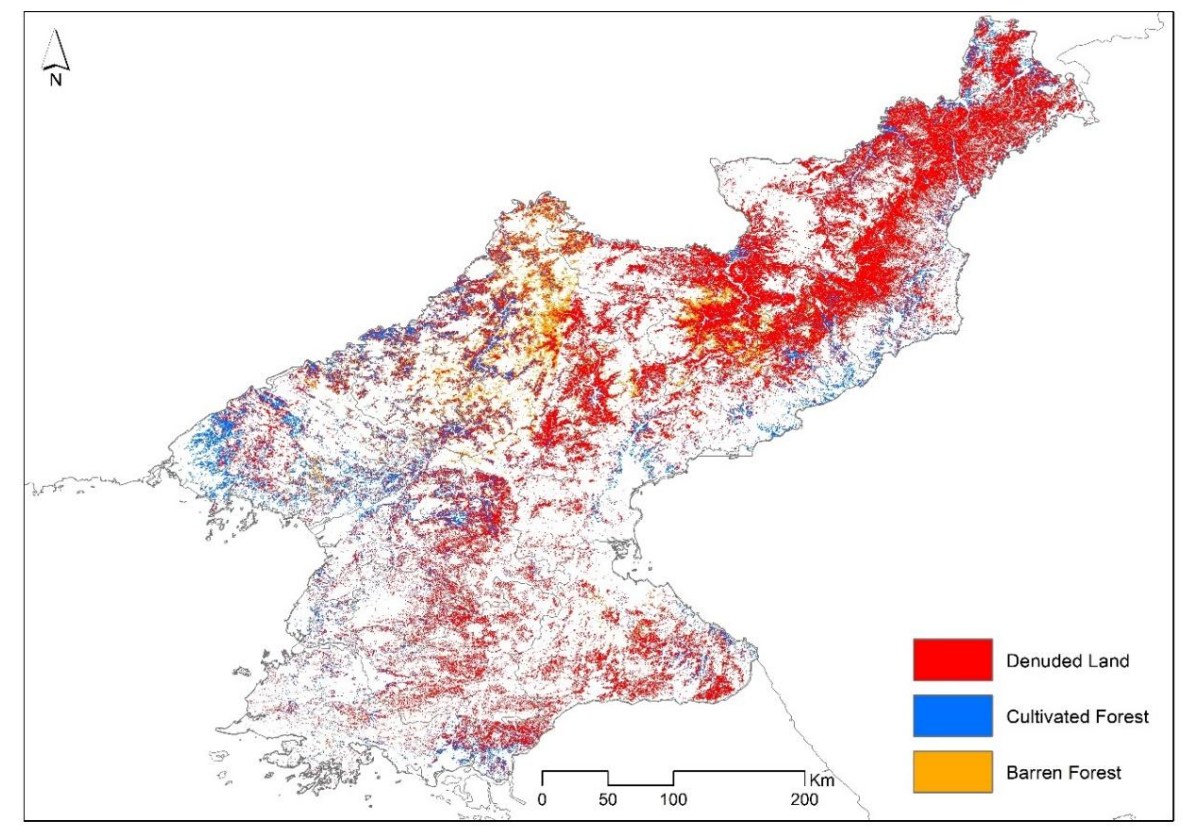

Figure 2. Classification map of degraded forest types in the DPRK. Data processed from a TerraSAR-X image, obtained 17 January 2014.

2.2.2. Identifying Suitable Regions Based on Appropriate Single Species for Afforestation/Restoration

The process for generating a spatial distribution map by species had two stages (Figure 3). First, suitable plant species were identified for restoration. Tree species suitability for planting were chosen 
based on three criteria: availability of species in South Korea, social demand in the DPRK and planting experience in the DPRK. The social demand in the DPRK indicates tree species which are economically valuable, but not ecologically suitable. Second, the potential distribution was identified for each species. Eleven variables, including climate, topography, and soil conditions, were reclassified as having scores between 1 and 5 (Table 1).

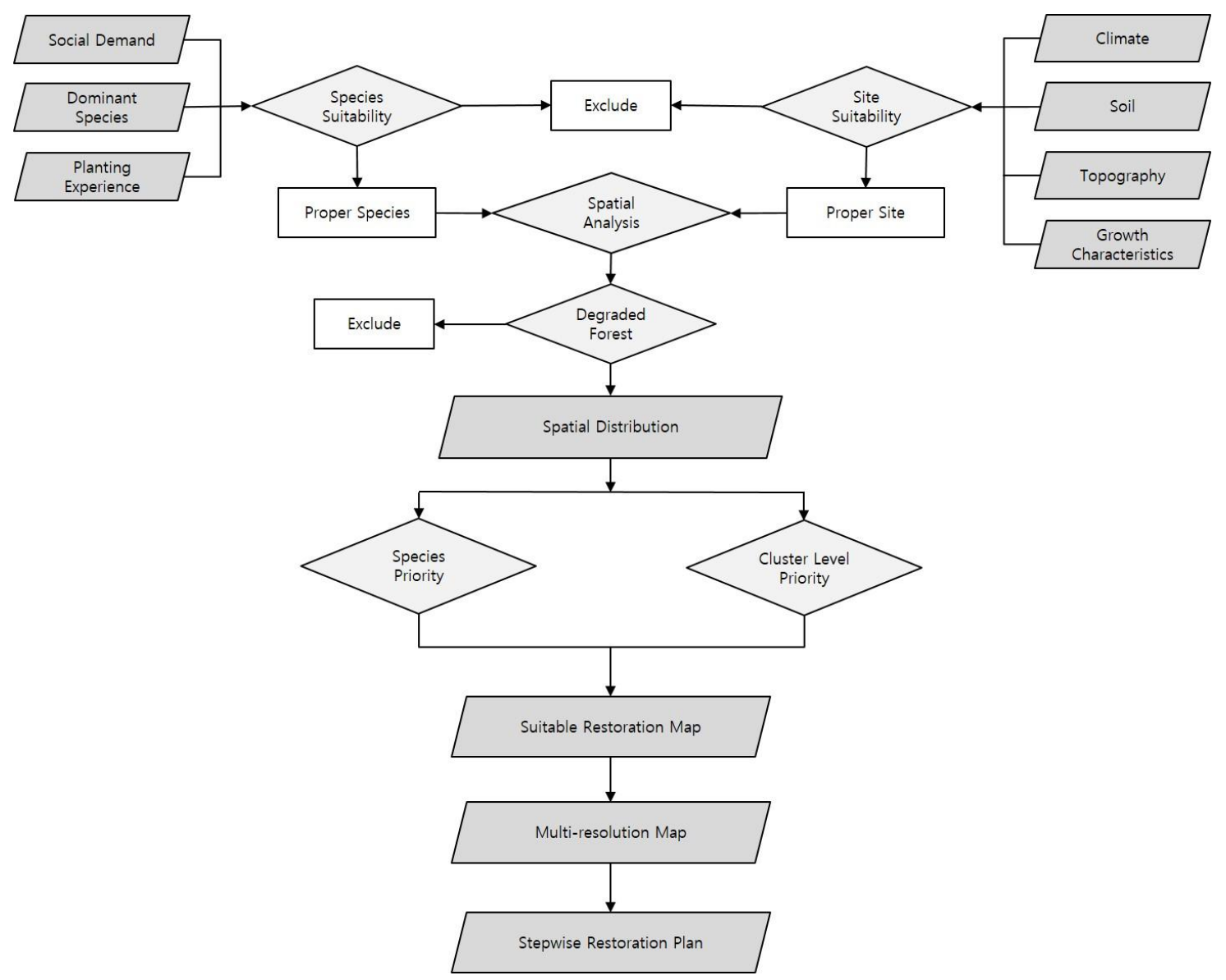

Figure 3. Research flow to establish a restoration plan in the DPRK.

Table 1. Example score card for plant distribution.

\begin{tabular}{|c|c|c|c|c|c|c|}
\hline Score & Climatic Zone & Elevation (m) & Aspect & Slope $\left({ }^{\circ}\right)$ & Topography & $\begin{array}{l}\text { Hardness } \\
\left(\mathrm{kg} / \mathrm{cm}^{2}\right)\end{array}$ \\
\hline 5 & middle & $200-400$ & $\mathrm{~S}$ & $<15$ & piedmont & $<0.5$ \\
\hline 4 & south & $<200$ & W & $15-20$ & valley & $0.5-1$ \\
\hline 3 & $\mathrm{x}$ & $400-600$ & $\mathrm{E}$ & $20-25$ & hillside & $1-2$ \\
\hline 2 & north & $>600$ & $\mathrm{~N}$ & $>25$ & tiptop & $>2$ \\
\hline 1 & polar & - & - & - & -1 & - \\
\hline Score & Rocks & Soil Texture & $\begin{array}{l}\text { Soil Depth } \\
\text { (cm) }\end{array}$ & $\begin{array}{c}\text { Soil } \\
\text { Moisture }\end{array}$ & $\begin{array}{c}\text { Organic } \\
\text { Matter (\%) }\end{array}$ & \\
\hline 5 & $\begin{array}{l}\text { Sedimentary rock, } \\
\text { metamorphic rock }\end{array}$ & $\mathrm{SL}, \mathrm{L}$ & $>60$ & $\begin{array}{c}\text { Humid, } \\
\text { semi-humid }\end{array}$ & $>5$ & \\
\hline 4 & Plutonic rock & CL, SCL & & Semi-arid & - & \\
\hline 3 & $\begin{array}{l}\text { limestone, volcanic rock, } \\
\text { pyroclastic material }\end{array}$ & LS & $30-60$ & - & $2-5$ & \\
\hline 2 & 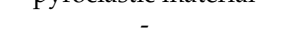 & S, SiL, SiCL & $<30$ & Arid & $<2$ & \\
\hline 1 & - & - & - & - & - & \\
\hline
\end{tabular}

Suitable environment ranges were based on accumulated data from South Korea [12-20,23,24], and assumed similar plant physiologic mechanisms across the Korean Peninsula. Classified areas with 
a score of 1 were excluded because they were considered an inappropriate area for planting. Except for the excluded regions, the plant growth and development maps were created by summing variables, resulting in a theoretical range from 11 to 55 . Total values in the 30-45 range were considered areas with the potential for high density trees.

\subsubsection{Identifying Priority Ranking for Afforestation/Restoration}

The distributions of total values provided a mechanism for prioritizing species and effectiveness in the integrated restoration map. When integrating, some discrimination was needed to choose the most suitable restoration tree. Species priority was determined based on suitable tree species for restoration in the DPRK provinces and suitable space for afforestation; site-specific suitable plants were given a weight of 2 for priority.

For spatial priority, natural distributions were given preference; the most common form was a congregated distribution [33]. The clustering shape was analyzed using Hot Spot Analysis [34] according to the Getis-Ord-Gi, a space-based analysis. Spatial groups in the DPRK were divided into Hot Spot and Cold Spot, and weighted by type. G-statistics represent a spatial autocorrelation index that is more suitable for discerning cluster structures of high or low concentration [35]. Z-score and $p$-value in the Getis-Ord-Gi were calculated in grid unit. Hot Spots were defined as high $z$-score regions with $p$-values $<0.05$, Cold Spots were defined as low $z$-score regions with $p$-values $<0.05$. Therefore, higher $z$-scores were correlated with higher cluster rates of distribution. In this study, to generate the integrated map, the cluster spatial priority was weighted for priority as follows: Hot Spot as 4, Not Significant as 2, and Cold Spot as 1.

$$
R S=\sum_{k=0}^{n}(P \times C)
$$

RS: Restoration Suitability, P: Order of Priority, C: Classification of Cluster

$$
\begin{gathered}
P=\operatorname{Max}(2 \times a \times S) \quad(\text { if target species }=\text { suitable }) \\
P=\operatorname{Max}(a \times S) \quad(\text { if } \text { target species } \neq \text { suitable })
\end{gathered}
$$

a: Hot Spot Classification (Hot Spot: 4, Not Significant: 2, Cold Spot: 1), S: Suitability Score

Single suitable region maps were combined using the weights from the priority ranking. A total value was calculated, and the highest value species were chosen for integrated spatial distribution.

\subsubsection{Optimal Spatial Level for Stepwise Afforestation/Restoration Plan}

We introduced a stepwise afforestation/restoration plan of degraded forest in the DPRK, specifically, general (long-term), detailed (medium-term), implementation (short-term) plans (Table 2). Maps with different spatial resolutions were prepared for each plan using spatial segmentation methods of block statistics. Block statistics are a type of neighborhood operation, where the local representative values have a certain level that can be extracted around a specific area [36]. Once a neighborhood is made, overlap is not allowed and all values are the same in the specific area. Therefore, this method can control the spatial scale with changing information within a grid. The $1 \mathrm{~km}$ and $12.5 \mathrm{~km}$ resolution scales were also chosen to agree with climate change scenarios generated by the Korea Meteorological Administration using HadGEM3-RA (Met Office, Devon, United Kingdom's) [37]. Agreement between climatic data and spatial scale satisfies the sustainable forest management plans in South Korea. Moreover, the maps are in general agreement with global trends in preparing adaptation plans [38]. The $5 \mathrm{~km}$ spatial resolution was chosen as an intermediate level between $1 \mathrm{~km}$ and $12.5 \mathrm{~km}$.

The restoration map with $12.5 \mathrm{~km}$ spatial resolution can be used for the general plan at the national level. Maps with $5 \mathrm{~km}$ and $1 \mathrm{~km}$ spatial resolutions can be used for detailed planning at the local level and implementation planning at the site level, respectively (Table 2). 
Table 2. Plan types and spatial resolution.

\begin{tabular}{cccc}
\hline Plan Type & Temporal Unit & Management Unit & Spatial Resolution \\
\hline General Plan & Long-term & National level & $12.5 \mathrm{~km} \times 12.5 \mathrm{~km}$ \\
Detailed Plan & Medium-term & Local level & $5 \mathrm{~km} \times 5 \mathrm{~km}$ \\
Implementation Plan & Short-term & Site level & $1 \mathrm{~km} \times 1 \mathrm{~km}$ \\
\hline
\end{tabular}

\section{Results}

\subsection{Suitable Tree Species for Afforestation/Restoration of DPRK's Forest}

The DPRK government announced a 10-year plan for reforestation [39], and recently released documents including the afforestation plan, major economic plant species, seedling production, and agroforestry plan. Furthermore, articles addressing forests are frequently published in the DPRK mass media, which indicates the level of interest in this subject. The 10-year plan (2001-2010) emphasized L. kaempferi, Pinus koraiensis Siebold and Zucc., Populus nigra var. italica Koehne, Robinia pseudoacacia L. [24], but in 2016, the major afforestation species also included L. kaempferi, P. koraiensis, Pinus rigida Mill., R. pseudoacacia, Acer saccharinum L., Populus euroamericana (Dode.) Guinier. South Korean non-governmental organizations supporting the DPRK reforestation have primarily focused on seven major plant species: P. koraiensis, L. kaempferi, Pinus strobus L., Pinus densiflora Siebold and Zucc., Quercus acutissima Carruth., Castanea crenata Siebold and Zucc., R. pseudoacacia. Based on the major species distribution, supported species in the DPRK, and the DPRK government demand, there are 23 tree species included in the reforestation plan: 11 coniferous trees and 12 deciduous trees (Table 3).

Table 3. List of suitable tree species for restoration of forests in the DPRK.

\begin{tabular}{|c|c|c|}
\hline Tree Type & Native Tree Species & Introduced Tree Species \\
\hline Coniferous Tree & $\begin{array}{c}\text {.Pinus densiflora Siebold and Zucc. } \\
\text {.Pinus koraiensis Siebold and Zucc. } \\
\text {.Larix olgensis var. koreana (Nakai) Nakai } \\
\text {.Abies holophylla Maxim. } \\
\text {.Abies nephrolepis (Trautv. ex Maxim.) Maxim. } \\
\text {.Picea jezoensis } \\
\text {.Pcea koraiensis Nakai } \\
\text {.Pinus thunbergii Parl. }\end{array}$ & $\begin{array}{l}\text {-Larix kaempferi (Lamb.) Carriere } \\
\text {.Pinus rigida Mill. } \\
\text { Pinus strobus L. }\end{array}$ \\
\hline Deciduous Tree & $\begin{array}{c}\text {-Quercus mongolica Fisch. ex Ledeb. } \\
\text {.Quercus acutissima Carruth. } \\
\text { ·Betula platyphylla var. japonica (Miq.) H. Hara } \\
\text { ·Fraxinus rhynchophylla Hance } \\
\text { ·Fraxinus mandshurica Rupr. } \\
\text {.Tilla amurensis Rupr. } \\
\text {.Zelkova serrate (Thunb.) Makino } \\
\text {.Castanea crenata Siebold and Zucc. } \\
\text { ·Juglans regia L. } \\
\text {.Populus nigra var. italic Koehne }\end{array}$ & $\begin{array}{l}\text { - Robinia pseudoacacia } \mathrm{L} \text {. } \\
\text {-Liriodendron tulipifera } \mathrm{L} \text {. }\end{array}$ \\
\hline
\end{tabular}

\subsection{Spatial Distribution Map of Suitable Tree Species}

Spatial distribution maps for each tree species were calculated based on 11 reclassified variables. Trees with a wide distribution include P. densiflora, P. koraiensis, Larix olgensis var. koreana (Nakai) Nakai, Quercus mongolica Fisch. ex Ledeb., Q. acutissima, and Picea jezoensis (Figure 4). There are differences based on data sources, but the results generally conform to the commonly dominant species in the DPRK. Each species has a different range and regional environmental factor affect score, particularly minimum and maximum values of the total score. In addition, there are areas with variables with a score of 1 categorized as inappropriate for planting. Using these factors, a characteristic species distribution was generated. 


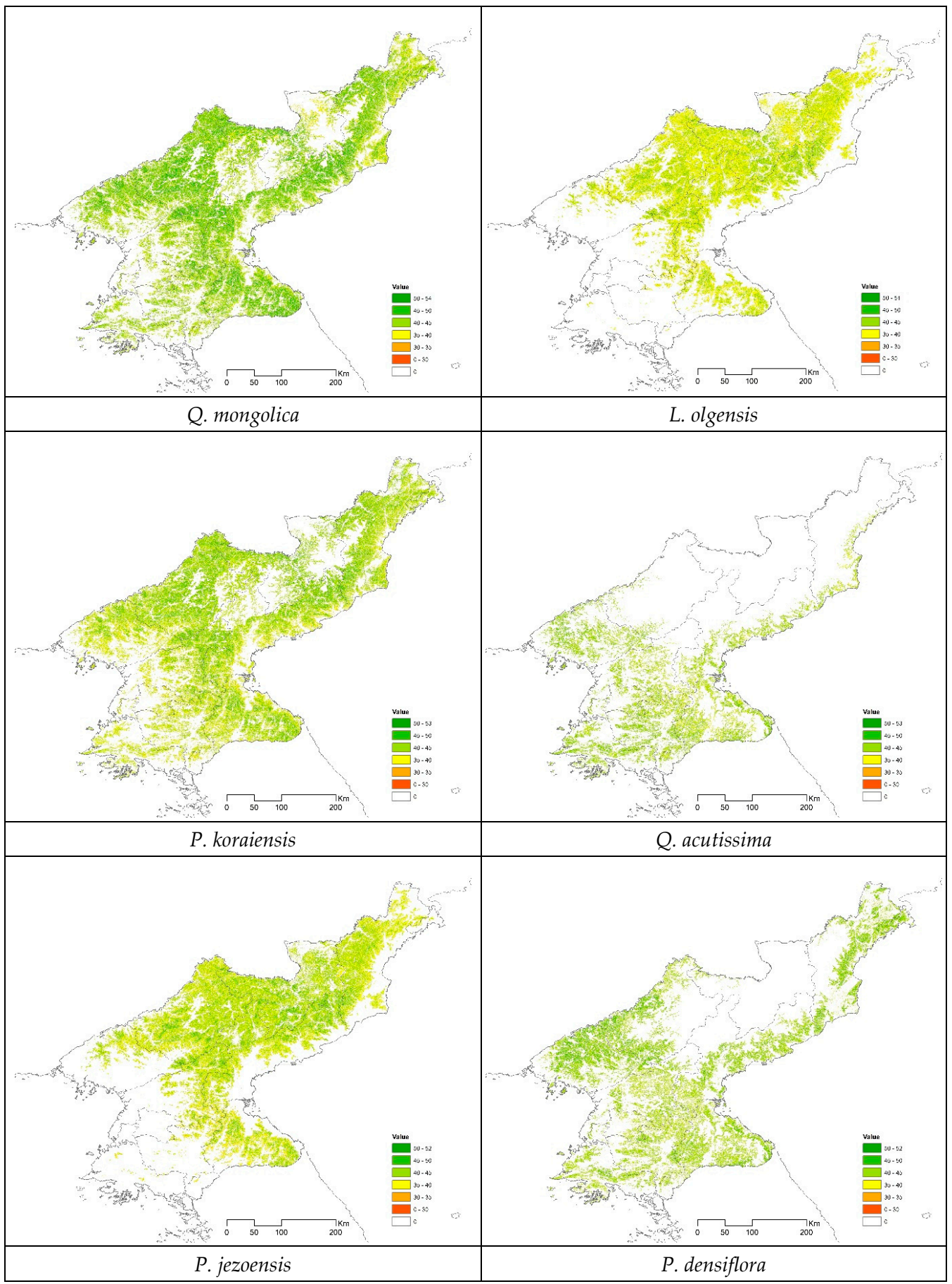

Figure 4. Spatial distribution map of suitable tree species.

\subsection{Suitable Tree Species for Degraded Forest in the DPRK}

\subsubsection{Suitable Tree Variety by Province in the DPRK}

Suitability maps from individual species information and their distribution were prepared by their order of priority. In this study, two types of priority were implemented: existing species within provinces and clustering distribution level in nature. First, a target area was identified. A region covered with green was not an urgent target, as this is considered a location with some reforestation progress in the DPRK, even if it is not ecologically superior. Urgent targets were considered vulnerable or degraded forest that needed restoration due to destroyed ecosystems. Degraded forest in the 
DPRK was identified from a TerraSAR-X image (17 January 2014), and consisted of denuded area $(2,670,000 \mathrm{ha})$, barren land (346,000 ha), and cultivated land (17,000 ha). These were respectively $30 \%, 4 \%$, and $0.2 \%$ of total mountainous region in the DPRK. Therefore, the most urgent restoration target areas were the denuded land. Restoration to denuded area in the DPRK will require high-effort human activity and should be treated as important because it takes a considerably larger area than other types of degraded forest. Planting denuded areas can achieve general afforestation without anti-erosion work [40]. A list of suitable tree species for denuded area in the DPRK provinces (Table 4) was compiled, based on site-specific data in the DPRK and conjugate species data from restorations in South Korea.

Table 4. List of suitable tree species for degraded forest in provinces of the DPRK.

\begin{tabular}{cc}
\hline Province & Suitable Major Species List \\
\hline HHN, HHS & P. densiflora, L. gmelinii, L. leptolepis, Q. mongolica, Q. acutissima \\
GW & P. densiflora, L. gmelinii, L. leptolepis, P. koraiensis, Q. mongolica, Q. acutissima \\
PAN, PAS & P. densiflora, L. leptolepis, P. koraiensis, Q. mongolica, Q. acutissima \\
JG & P. densiflora, L. gmelinii, L. leptolepis, P. koraiensis, Q. mongolica, B. platyphylla \\
YG & L. gmelinii, P. koraiensis, Q. mongolica, B. platyphylla \\
HGN, HGS & P. densiflora, L. gmelinii, L. leptolepis, P. koraiensis, Q. mongolica Q. acutissima, B. platyphylla \\
\hline
\end{tabular}

HHN: Hwanghaebuk-do, HHS: Hwanghaenam-do, GW: Gangwon-do, PAN: Pyeonganbuk-do, PAS: Pyeongannam-do, JG: Jagang-do, YG: Yanggang-do, HGN: Hamgyeongbuk-do, HGS: Hamhyeongnam-do.

\subsubsection{Spatial Priority Ranking of Suitable Tree Species}

Hot Spot Analysis was conducted to prioritize the cluster distribution in suitable spaces for each species. The results presented a range of suitable plant distributions with z-score for the clustering region; independent variables excluded a score of 1 within each grid. Regions that appeared sporadic were classified as Cold Spots. Figure 5 reflects the concentration and dispersion for suitable distribution patterns.

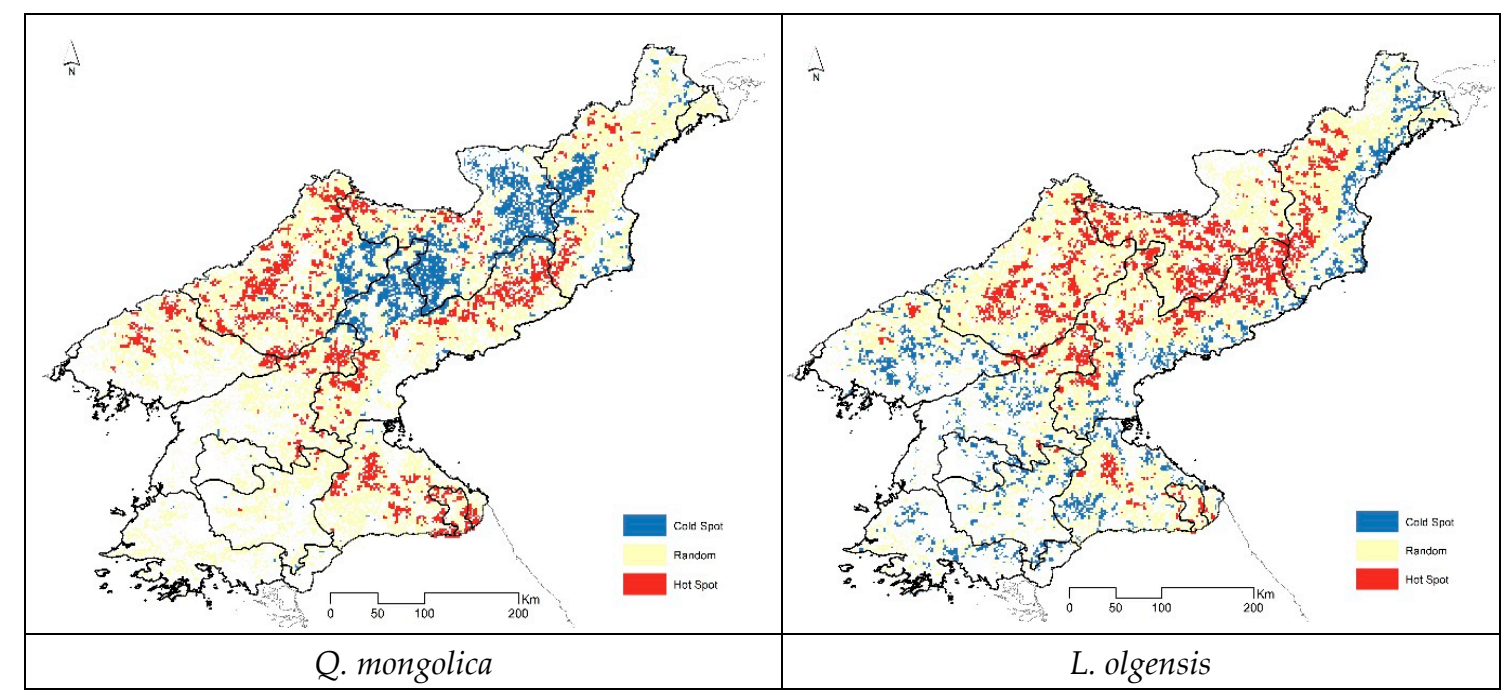

Figure 5. Cont. 


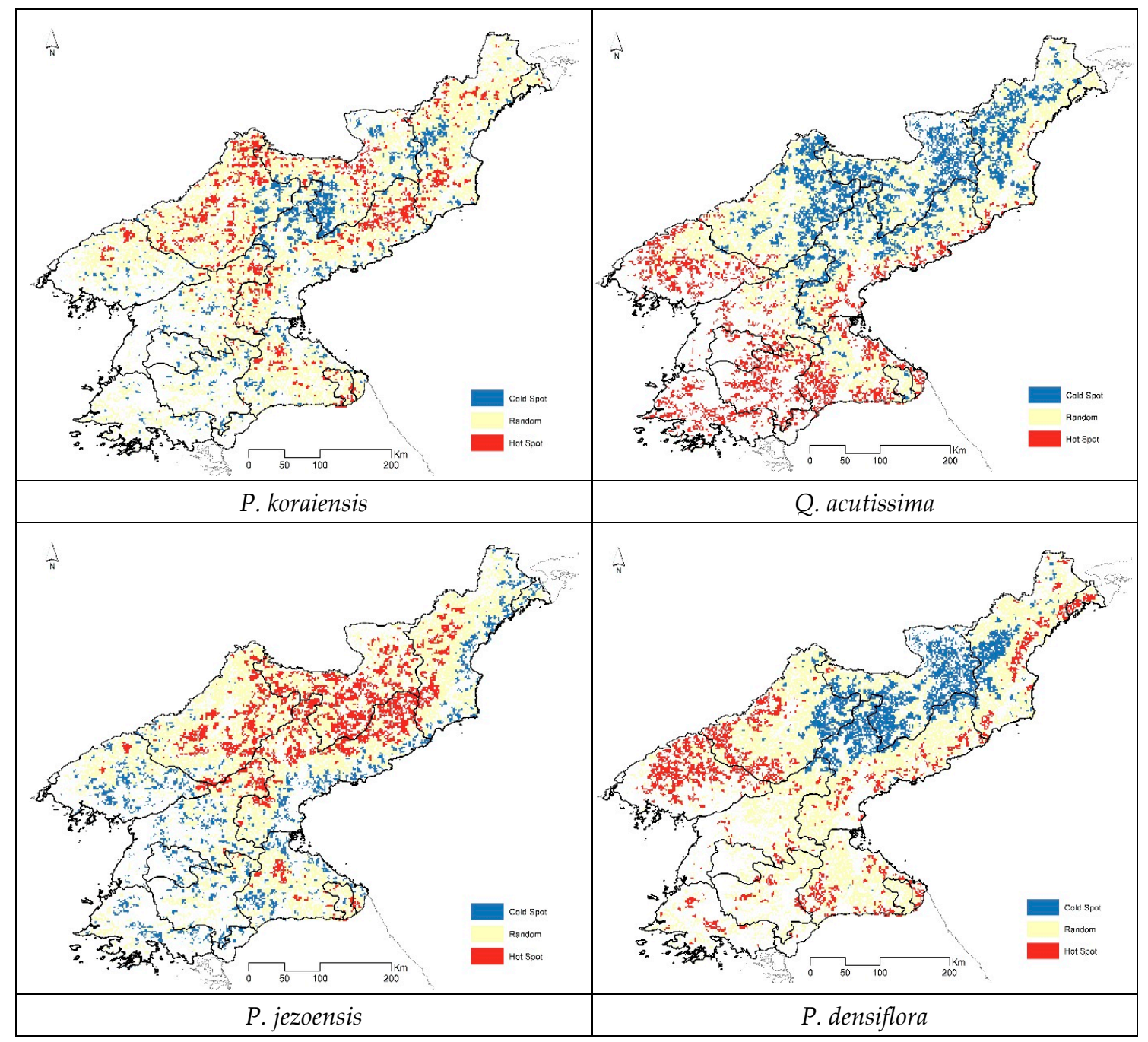

Figure 5. Spatial priority ranking of suitable tree species.

\subsection{Spatial Distribution Map of Suitable Tree Species}

The spatial distribution map of suitable tree species was prepared by prioritizing species based on growth and development scores, suitable species to restore denuded land, and clustering rate. As shown in Figure 6, P. jezoensis occupied the largest area, followed by Q. mongolica, L. olgensis, P. koraiensis, Q. acutissima, and P. densiflora. Species with smaller distributions included Pinus thunbergii Parl., Zelkova serrate (Thunb.) Makino, B. platyphylla, Abies nephrolepis (Trautv. Ex Maxim.) Maxim., R. pseudoacacia, Fraxinus rhynchophylla Hance, Larix kaempferi (Lamb.) Carriere, Fraxinus mandshurica Rupr., P. rigida, C. crenata, Picea koraiensis Nakai, Liriodendron tulipifera L., Abies holophylla, P. nigra, Tilla amurensis Rupr. Furthermore, $P$. strobus and Jglans regia L. were not included in the integrated map because they have different priorities to the other species. 


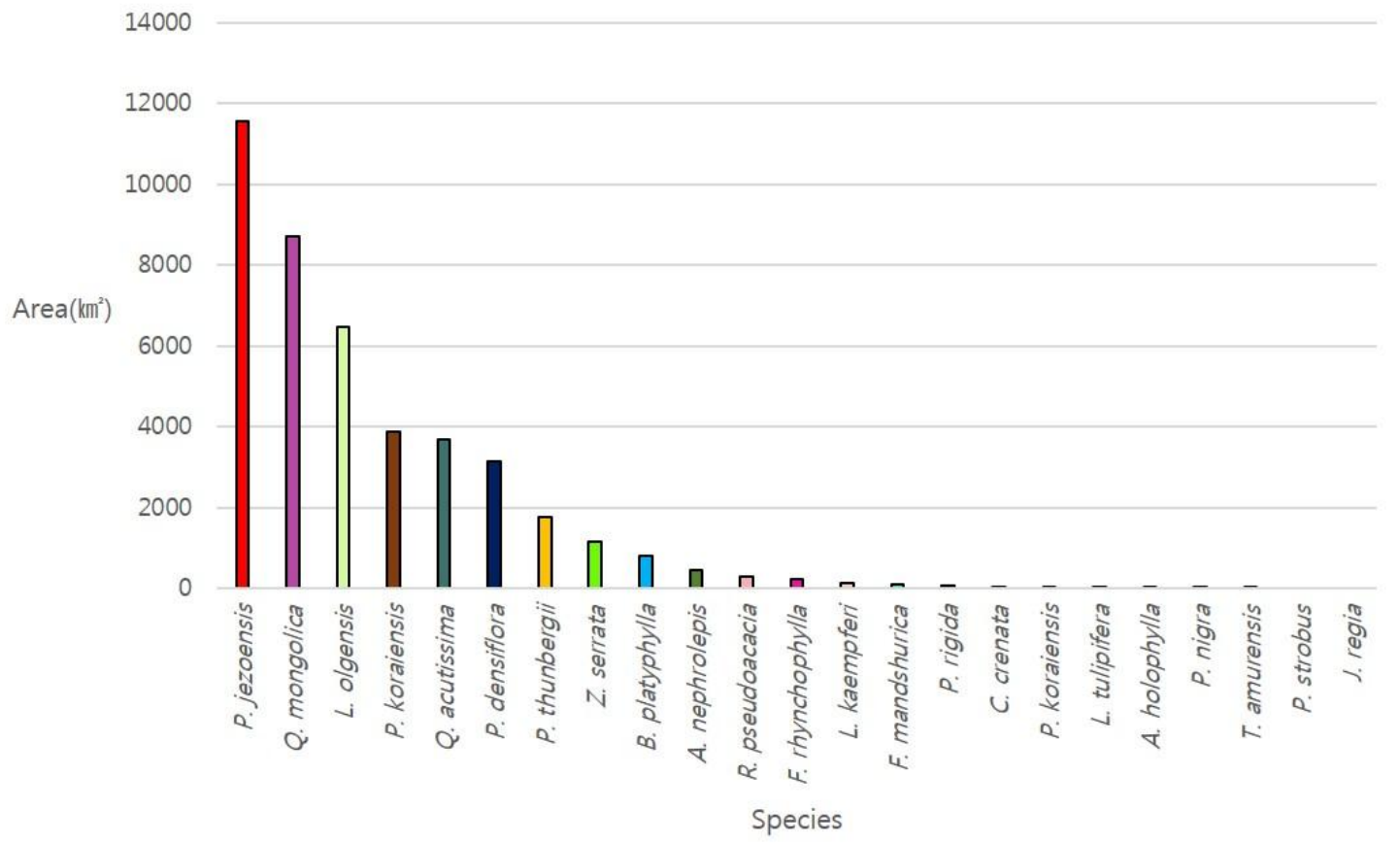

Figure 6. Extent of restoration by species in degraded forest in the DPRK.

The spatial patterns in the restoration map for degraded forest in the DPRK (Figure 7) show that P. densiflora, Q. mongolica, and L. olgensis have a complex distribution. P. thunbergii and Q. acutissima are found in the low land near the eastern coast of Korea, P. densiflora is densely distributed northwards from the middle of HGN province. L. olgensis is mainly found in the inland region in western HGN province near YG province. Upland HGS province has a similar species construction as HGN province, while the lowland closed coast has a more definitely divided sporadic distribution. YG province is dominated by L. olgensis and P. jezoensis, and there is particularly scant afforestation near Baekdusan Mt. and high elevation zones, due to the absence of degraded forest. Multiple species are distributed in JG province, primarily the largest variety species. P. jezoensis, Z. serrate, P. densiflora, Q. mongolica, L. olgensis, P. koraiensis, A. nephrolepis, B. platyphylla cover wide areas. P. densiflora is clustered over the middle and western part of JG province. In PAN province, P. jezoensis, Q. mongolica, P. densiflora are distributed inland near China, but $Q$. acutissima, $P$. thunbergii, $Z$. serrate dominate the outermost regions. PAS province has district species compositional differences between the east and west. As high elevation and relative cold climate in the eastern part, P. jezoensis, Q. mongolica, and P. koraiensis are located. Q. acutissima dwindle in distribution from the center to the borders of PAS province. Western PAS province has sporadic distribution of $P$. thunbergii, Z. serrate, and Q. acutissima, because it is a plains region which decreases the amount of degraded forest. P. thunbergii, Z. serrate, and Q. acutissima are mainly distributed in HHN and HHS provinces, and degraded forest appears diffuse. The high mountainous area (Baekdudaegan) in GW province has the most intricate tree distribution in southern DPRK, with mixed species from upland and lowland. The major species in GW province are P. jezoensis, Z. serrate, Q. acutissima, P. densiflora, Q. mongolica, P. koraiensis, Fraxinius mandshurica Rupr., F. rhynchophylla. Finally, P. thunbergii and Q. acutissima are found on the eastern coast of Korea. 


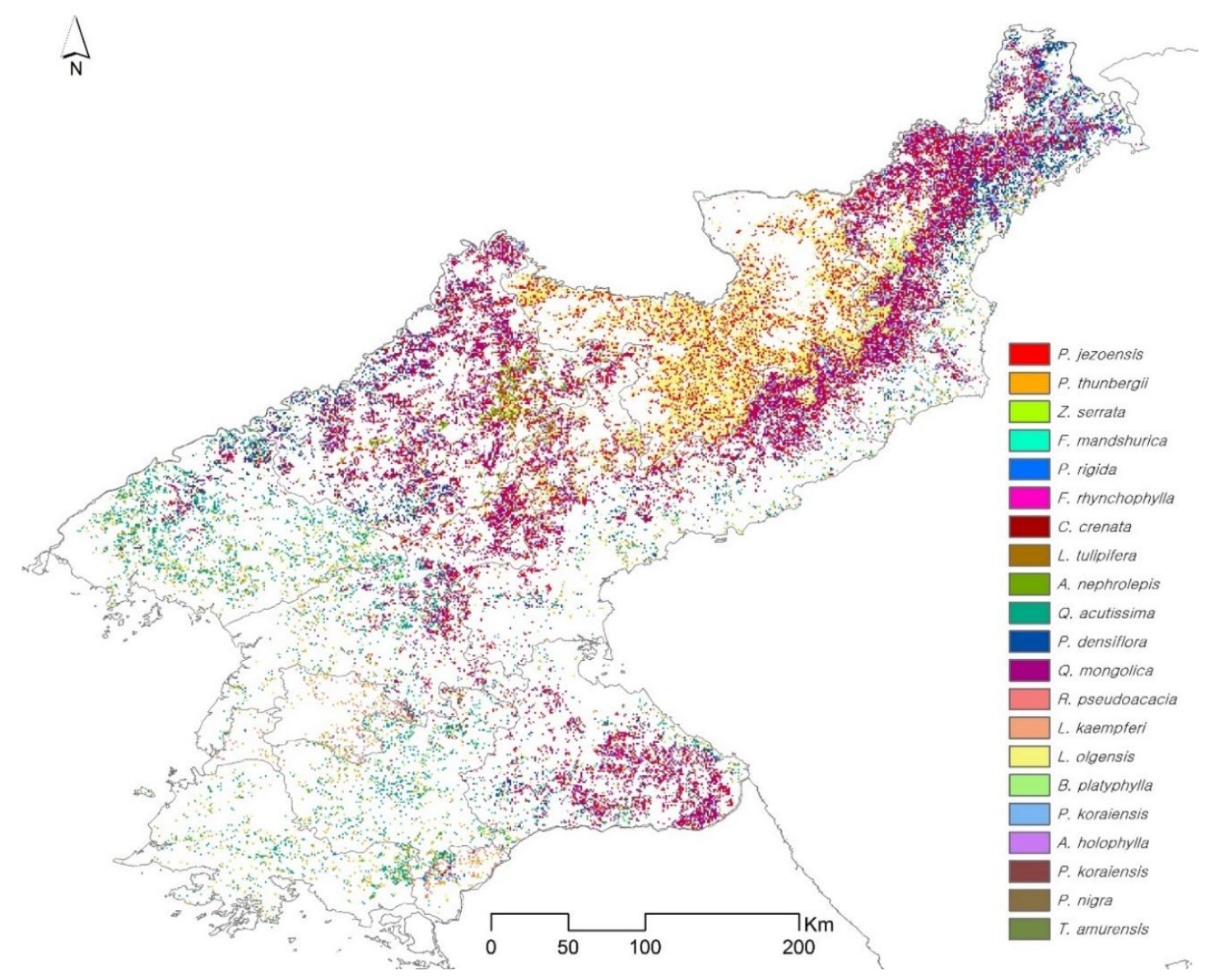

Figure 7. Spatial distribution map of suitable tree species in the DPRK.

\subsection{Application: Stepwise Afforestation/Restoration Plan for Degraded Forest in the DPRK}

Zoning based on suitable species supports decision making for restoration of degraded forest in the DPRK. The spatial distribution map for suitable restoration provides concrete spatial information, and is detailed enough to provide forest management guidelines for all of the DPRK. It is impossible to restore all degraded forest in the DPRK at once. Therefore, several steps are needed, which should start with a risk management plan during the preparatory stage. A flexible stepwise plan by period or level should be created, for example, a general plan (long-term), detailed plan (medium-term), and implementation plan (short-term). In this study, a forest restoration scenario using a spatial segmentation method is suggested to support decision making at the different plan levels. This is based on the spatial distribution map of suitable restoration for degraded forest in DPRK (Figure 5). The long-term general plan map has a $12.5 \mathrm{~km}$ resolution appropriate for planning at the national level. It provides general guidance on the distribution pattern of suitable species. The medium-term detailed plan has a $5 \mathrm{~km}$ grid size, with technical data to aid decision making at the province or local level. Finally, the short-term implementation plan with the $1 \mathrm{~km}$ resolution provides the most detail to implement afforestation services at specific sites (Figure 8) and includes a field survey. The results presented in the 5 and $12.5 \mathrm{~km}$ resolution maps used Block Statistics, a type of neighborhood operations to show general patterns and simplify of the species distributions compared with $1 \mathrm{~km}$. The number of major species depends on the specific resolution, there are 21 in the $1 \mathrm{~km}$ resolution map, 16 in the $5 \mathrm{~km}$ resolution map, and 12 in $12.5 \mathrm{~km}$ resolution map. 


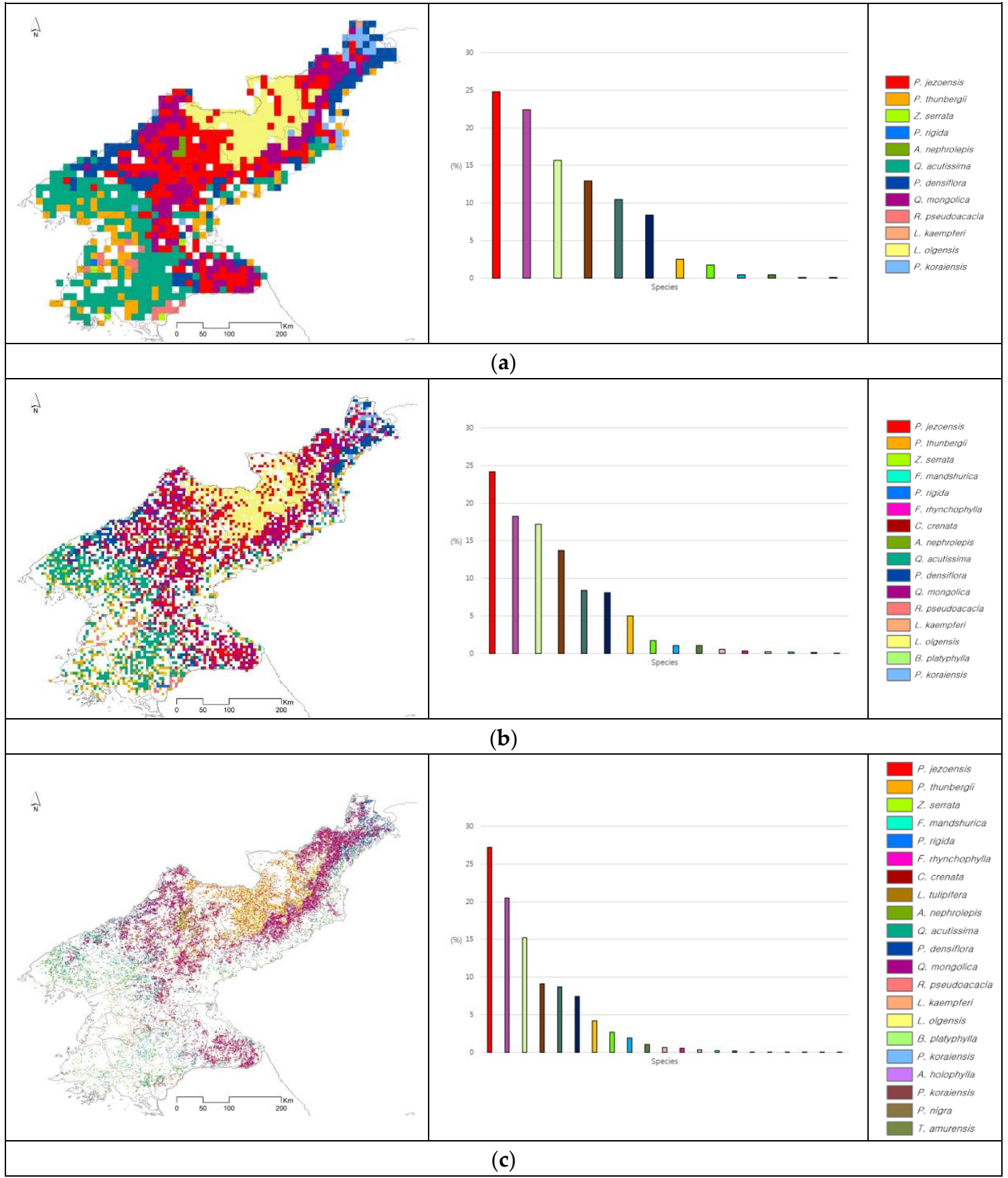

Figure 8. Stepwise restoration plan for degraded forest with different map resolutions. (a) National Plan, General Plan, Long-term, $12.5 \mathrm{~km} \times 12.5 \mathrm{~km}$; (b) Regional Plan, Detailed Plan, Mid-term, $5 \mathrm{~km} \times 5 \mathrm{~km}$; (c) Site-specific Plan, Implementation Plan, Short-term, $1 \mathrm{~km} \times 1 \mathrm{~km}$.

\section{Discussion}

There is an ecological connection between North and South Korea, but the status of their forest ecosystems may differ slightly. Forests in the DPRK have gradually been degraded and have created a devastating problem, despite national efforts. It is not easy to obtain data or data collection techniques utilized in field surveys because of political conditions. This is clearly problematic, as such data and research methods are essential for predicting data and generating models. However, there is political pressure in South Korea to prepare and implement a forest restoration plan in North Korea. The most important issue in addressing forest degradation is intensifying the weakened forest function created by 
farmland expansion and soil loss across the DPRK due to financial stress [41]. The distribution patterns of the main species in the DPRK must be understood to achieve healthy forests. The reforestation sequence needs guidelines at all levels: Middle level guidelines for managing degraded forests at the provincial or local level, and a detailed restoration map to create a corresponding implementation plan. This type of data, combining on-site studies and the distribution map of each species, can be used to create flexible policies.

\section{Conclusions}

This study has identified suitable species and introduced a stepwise afforestation plan for restoring denuded forest, according to the prioritization of degraded forest areas in the DPRK. The research results show that there are 12 suitable afforestation tree species based on social demand and natural conditions. These tree species include 11 coniferous and 12 deciduous trees, with 5 introduced tree species that the DPRK would like to set up. The ranges in growth and development for each tree species were calculated from 11 variables, including climate, topography and soil factors; consequently, the distribution of tree species was calculated from referenced DPRK data. Species were identified based on site-specific properties, which resulted in varying distribution patterns. To generate the suitability map, weighting or prioritization of species and spatial concentrations of suitable area were used. P. jezoensis has the largest suitable area, followed by Q. mongolica, L. olgensis, P. koraiensis, $Q$. acutissima, and P. densiflora. Three resolutions were used to prepare maps for stepwise restoration plan: the 12.5, 5, and $1 \mathrm{~km}$ spatial resolution match different plan levels for general (national level), detailed (local level), implementation (site level) planning. With the help of the multi-spatial resolution maps, the stepwise restoration plan can be made and applied for different levels of decision making from national, local to site.

Acknowledgments: This study was supported by "The Generation of Climate Change Information for RCP-based climate data for the Assessment of Impact on various measures (KMIPA2015-2130)" provided by Korea Meteorological Administration (Korea Meteorological Institute) and "R\&D Program for Forest Science Technology (2017044B10-1819-BB01)" provided by Korea Forest Service (Korea Forestry Promotion Institute).

Author Contributions: Sle-gee Lee and Woo-Kyun Lee wrote the paper. Hyun-Ah Choi analyzed the results. Hyeji Yoo, Cholho Song, and Sungeun Cha contributed in data processing. Sang-Won Bae, Yowhan Son, and Woo-Kyun Lee gave advice and overall comments.

Conflicts of Interest: The authors declare no conflicts of interest

\section{References}

1. Goh, K. Green Korea Project-Afforestation experience of South Korea and afforestation scheme for North Korea. In Proceedings of the 1st International Symposium in Commemoration of the Foundation of Green Asia Organization, Seoul, Republic of Korea, 19 March 2014.

2. Xu, J.C.; van Noordwijk, M.; He, J. Participatory agroforestry development for restoring degraded sloping land in DPR Korea. Agr. Syst. 2012, 85, 291-303. [CrossRef]

3. Kang, S.; Choi, W. Forest cover changes in North Korea since the 1980s. Reg. Environ. Chang. 2014, 14, 347-354. [CrossRef]

4. Park, K. Forest condition and policy in North Korea. In KREI Quarterly Agricultural Trends in North Korea; KREI: Naju, Republic of Korea, 2013; Volume 15, pp. 1-21.

5. Byeon, J. Forest Change in the DPRK and Strengthen Ability for Recovery; KAST: Seongnam, Republic of Korea, 2014.

6. Moon, K.H.; Park, D.K. The role and activities of NGOs in reforestation in the northeast Asian region. For. Ecol. Manag. 2004, 201, 75-81. [CrossRef]

7. Zomer, R.J.; Trabucco, A.; Bossio, D.A.; Verchot, L.V. Climate change mitigation: A spatial analysis of global land suitability for clean development mechanism afforestation and reforestation. Agric. Ecosyst. Environ. 2008, 126, 67-80. [CrossRef]

8. Bae, J.S. A preliminary review of REDD mechanism for rehabilitating forest degradation of North Korea. J. Korean For. Soc. 2013, 102, 491-498. [CrossRef] 
9. Park, M.S.; Lee, H. Forest policy and law for sustainability within the Korean Peninsula. Sustainability 2014, 6, 5162-5186. [CrossRef]

10. Bae, J.S.; Joo, R.W.; Kim, Y. Forest transition in South Korea: reality, path and drivers. Land Use Policy 2012, 29, 198-207. [CrossRef]

11. Democratic People's Republic of Korea. Tree Nursery Reference Book for Laborer; North Korea Industrial Publishing: Pyeongyang, Democratic People's Republic of Korea, 2016.

12. Korea Forest Research Institute. Forest, Forestry and Dominant Species In North Korea; Korea Forest Research Institute: Seoul, Republic of Korea, 2004.

13. Korea Forest Research Institute. Forest, Forestry and Dominant Species in North Korea (II); Korea Forest Research Institute: Seoul, Republic of Korea, 2005.

14. Korea Forest Research Institute. Forest, Forestry and Dominant Species in North Korea (III); Korea Forest Research Institute: Seoul, Republic of Korea, 2006.

15. Korea Forest Research Institute. Forest, Forestry and Dominant Species in North Korea (IV); Korea Forest Research Institute: Seoul, Republic of Korea, 2007.

16. Korea Forest Research Institute. Desired Tree Species In Korea; KFRI: Daejeon, Republic of Korea, 2007.

17. Korea Forest Research Institute. Forest, Forestry and Dominant Species in North Korea; KFRI: Daejeon, Republic of Korea, 2009; p. 367.

18. Korea Forest Research Institute. History and Application of Right Tree on Right Site; KFRI: Daejeon, Republic of Korea, 2009.

19. Korea Forest Research Institute. Forest, Forestry and Dominant Species in North Korea; KFRI: Daejeon, Republic of Korea, 2011.

20. Korea National Arboretum. 2015 Report of Conservation Project of Threatened Plants for Climate Change; Korea National Arboretum Director General: Pocheon, Republic of Korea, 2015.

21. Korea Forest Service. Forestry Statistics Annual Report; Korea Forest Service: Daejeon, Republic of Korea, 2015.

22. Korea Forest Service. Handbook of Forest Cooperation between South and North Korea; Korea Forest Service: Daejeon, Republic of Korea, 2016.

23. Korea Forest Service. Comprehension of Dominant Species in North Korea; Korea Forest Service: Daejeon, Republic of Korea, 2016.

24. The Korean Academy of Science and Technology. Report of Forest Ecology and Afforestation Species Suitable Environmental Change; KAST: Seongnam, Republic of Korea, 2014.

25. Yim, Y. Distribution of forest vegetation and climate in the Korean Peninsula III. Distribution of tree species along the thermal gradient. Jpn. J. Ecol. 1977, 27, 177-189.

26. Javis, A.; Reuter, H.I.; Nelson, A.; Guevara, E. Hole-Filled SRTM for the Globe Version 3, CGIAR-CSI SRTM 90m Database. Available online: http:/ / srtm.csi.cgiar.org. (accessed on 2 December 2017).

27. An, S.; Park, S.; Kim, C. Chosun Geographical Compendium (Soil Geography). (Democratic People's Republic of Korea); DPRK: Pyeongyang, Democratic People's Republic of Korea, 1978.

28. Park, S.J.; McSweeney, K.; Lowery, B. Prediction of soils using a process based terrain characterization. Geoderma 2001, 103, 249-272. [CrossRef]

29. Sörensen, R.; Zinko, U.; Seibert, J. On the calculation of the topographic wetness index: evaluation of different methods based on field observations. Hydrol. Earth Syst. Sci. Discuss. 2006, 10, 101-112. [CrossRef]

30. Passioura, J.B. Soil conditions and plant growth. Plant Cell Environ. 2002, 25, 311-318. [CrossRef] [PubMed]

31. Korea Forestry Promotion Institute. Analyzing Forest Condition in the DPRK Utilized Satellite Data; Kofpi: Seoul, Republic of Korea, 2015.

32. Kim, E.; Lee, S.; Cho, H. Segment-based land cover classification using texture information in degraded forest land of North Korea. Korean J. Remote Sens. 2010, 26, 477-487.

33. Cox, G. General Ecology Laboratory Manual, 8th ed.; McGraw-Hill: New York, NY, USA, 2001.

34. Chang, B.A.; Pearson, W.S.; Owusu-Edusei, K., Jr. Correlates of country-level nonviral sexually transmitted infection hot spots in the US: application of hot spot analysis and spatial logistic regression. Ann. Epidemiol. 2017, 27, 231-237. [CrossRef] [PubMed]

35. Manepalli, U.R.; Bham, G.H.; Kandada, S. Evaluation of hotspots identification using kernel density estimation $(\mathrm{K})$ and Getis-Ord $\left(\mathrm{Gi}^{*}\right)$ on I-630. In Proceedings of the 3rd International Conference on Road Safety and Simulation, Indianapolis, IN, USA, 14-16 September 2011. 
36. Kwon, S.; Joo, W.; Kim, W.; Kim, J.; Kim, E. Analyzing site characteristics and suitability for wind farm facilities in forest lands. J. Korean Assoc. Geogr. Inf. Stud. 2014, 17, 86-100. [CrossRef]

37. Gu, H.; Yu, Z.; Yang, C.; Ju, Q.; Yang, T. High-resolution ensemble projections and uncertainty assessment of regional climate change over China in CORDEX East Asia. Hydrol. Earth Syst. Sci. 2017. [CrossRef]

38. Korea Forestry Promotion Institute. Selecting Suitable Afforestation Species and Upgrading Mountainous Soil in the DPRK; Kofpi: Seoul, Republic of Korea, 2016.

39. Kim, S.; Park, S.; Park, K. A study on new change of forest management in the DPRK by introducing agroforestry of sloping land management. Inst. Peace Aff. 2016, 28, 127-157.

40. Korea Forest Research Institute. Master Plan of Forest Science Research to Respond New Climatic System (2016-2020); KFRI: Daejeon, Republic of Korea, 2016.

41. Park, K.; Lee, S.; Park, S. A study on the North Korea's change of forest policy since the economic crisis in 1990's. Inst. Peace Aff. 2009, 21, 459-492.

(C) 2018 by the authors. Licensee MDPI, Basel, Switzerland. This article is an open access article distributed under the terms and conditions of the Creative Commons Attribution (CC BY) license (http://creativecommons.org/licenses/by/4.0/). 